\title{
Fractal characteristics of mudstone microscopic morphology in MATLAB environment
}

\author{
HUANG Dongmei ${ }^{1}$, ZHANG Zhenquan ${ }^{1}$, LIN Xiaofei ${ }^{2}$, LI Huaxue ${ }^{1}$ \\ 1,College of Mining and Safety Engineering, Shandong University of Science \\ and Technology, Qingdao, China 266590; 2, Civil Engineering and Agriculture \\ School, Anhui University of technology, Ma'anshan, China, 243002.
}

\begin{abstract}
By using the image processing and numerical functions of MATLAB, after the treatment of SEM image to mudstone specimen with threshold segmentation, filtering and other procedures, we can obtain binary image of mudstone; and determine the fractal dimension of the image by MATLAB program. The results showed that: MATLAB fractal image processing is simple and convenient, the computation of regular fractal dimension by using Koch curve showed that the calculating results in this way are accurate and reliable. The calculation of the mudstone fractal dimension showed that mudstone has different fractal dimension under different intensities. On the whole, the bigger the intensity, the smaller the fractal dimension.
\end{abstract}

Keywords : mudstone microscopic morphology; image processing; fractal dimension

\section{Introduction}

Fractal geometry and fractal dimension were formally put forward by a French mathematician, $\quad$ B.B.Mandelbrot ${ }^{[1]}$. Fractal geometry is the discipline which regards irregular geometry as its research object. It can deeply describes the form which seems disorganized in nature ${ }^{[2]}$. Starting with natural geometry, then it is extended to the field of biology, materials science, earth science, etc. The fractal has the following characteristics: As a whole, fractal geometric graph is irregular everywhere; specific to each section, it has some similarity with the entirety. Its main value lies in that it provide a possibility between extreme order and the real chaos, which supply the efficient method to solve complex geometry problems that are hard to deal with

2 Fractal dimension and application in rock microscopic morphology analysis

Dimension of fractal geometric graph is called fractal dimension for short, which is an important tool to describe fractal complex structure. Nowadays there are multiple definitions and calculation methods of multi-fractal dimension which mainly include Hausdroff dimension, box counting dimension, information dimension, capacity dimension, interrelation dimension and so on. Among them, Hausdroff dimension is the basis of fractal theory, which was mainly used for theoretical derivation of fractal geometry, but is useless in the actual application problem of fractal geometry. Box dimension is relatively simple in its mathematical calculations and its physical meaning is more intuitive, and is easily to be calculated procedurally, so it is widely used in calculation and practice of the fractal dimension. 
In recent years, fractal has been applied to many subjects. It is important not only in theory but in practice. Feranie $\mathrm{S}$ and other foreign scholars $^{[6]}$ calculated the fractal dimension of rock samples by scanning the rock slices; Jiande Li and others $^{[7]}$ analyzed granite rock burst particulate debris under uniaxial and triaxial compression conditions with fractal theory then got a conclusion that clastic rock burst owns fractal characteristics; Liu Jinghong and others ${ }^{[8]}$ did fractal research about the process that rock materials' crack expand to rupture, which revealed microscopic fracture mechanism of crack from start, expansion to perforation and also proved that crack propagation process inside the material owns fractal characteristics, it also proved that destruction and instability of rock fracture is closely related to the distribution, start, expansion and perforation of microcracks inside .

A large number of studies showed that: The fractal dimension is a good parameter in expressing the complexity of rock structure, however previous studies focused more on the macroscopic fracture structure and less on calculating the fractal dimension of microscopic cracks. So this article focuses on fractal study of rock microscopic morphology.

3 Fractal dimension calculation of mudstone microstructure morphology 3.1 Analysis of mesoscopic morphology for mudstone

Take 3 mudstone as specimens, the secondary electron imaging were got by SEM, each sample obtain 6 images under different multiples, for respectively 100 times, 200 times, 500 times, 1000 times, 2000 times and 5000 times, respectively, and calculated the fractal dimension under various multiples. Six multiples of the SEM images are shown in figure 1 for specimen 1\# (for example), the electronic intensity inconsistencies result in the different brightness in figure caused by the uneven surface.

We obtained some information from the above photos: the mesoscopic structure of mudstone is relatively complex, mudstone present irregular scaly aggregation, individual place appears irregular Or angular lamellar stratification with some cavities and cracks, and the

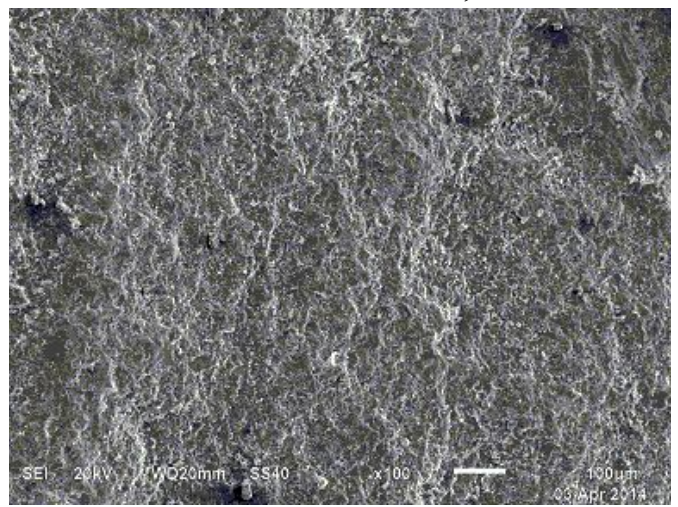

(a) $100 \mathrm{X}$

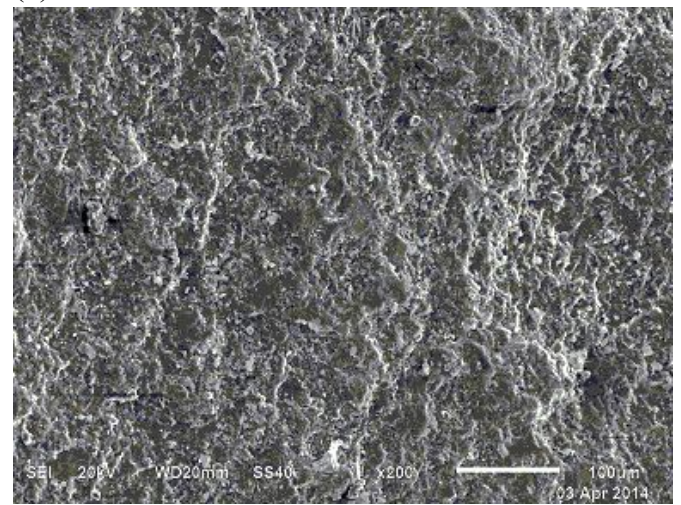

(b) $200 \mathrm{X}$

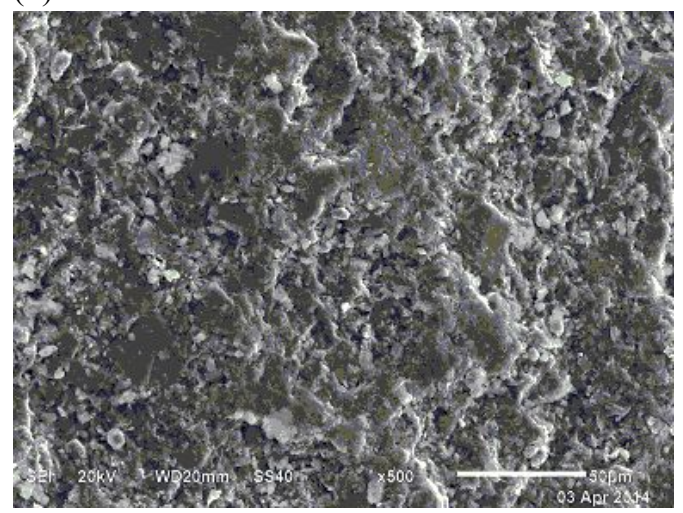

(c) $500 \mathrm{X}$ 


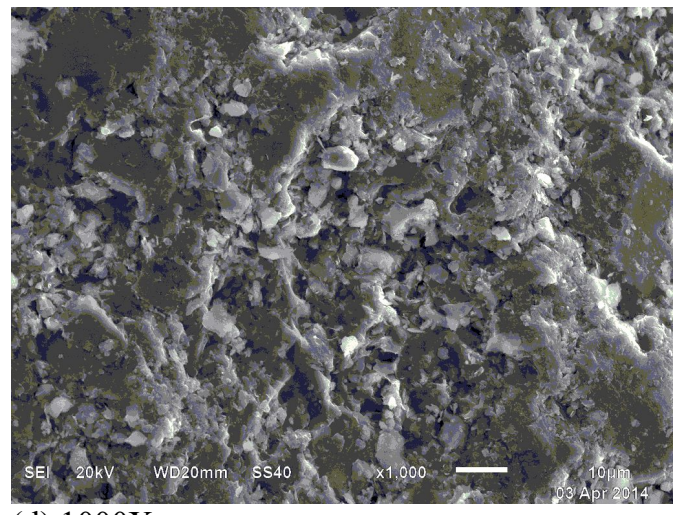

(d) $1000 \mathrm{X}$

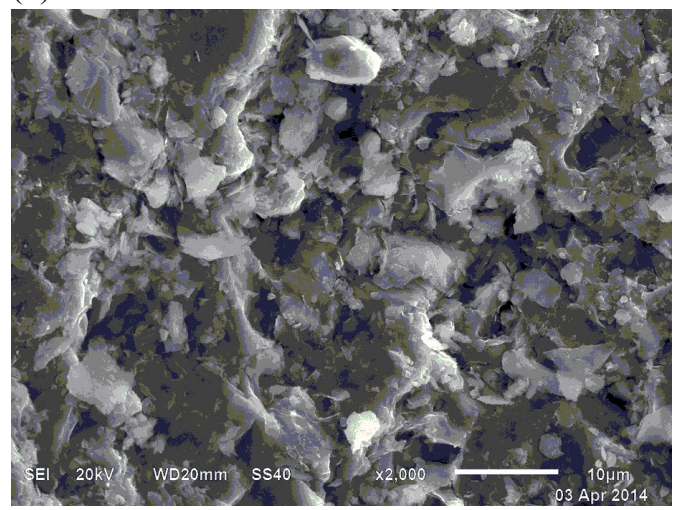

(e) $2000 \mathrm{X}$

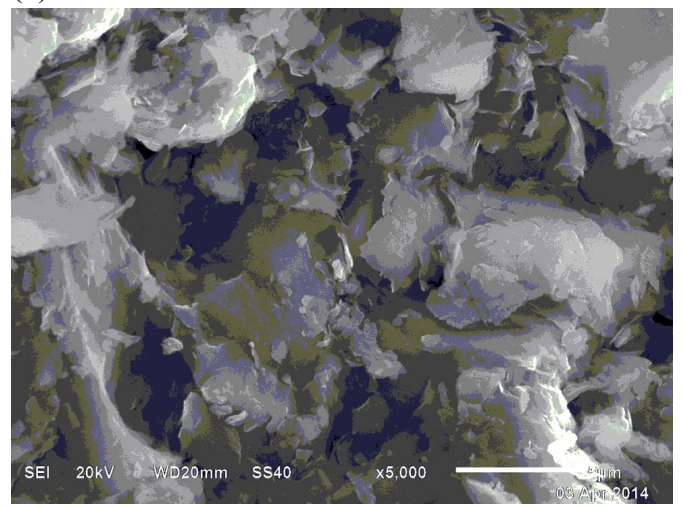

(f) $5000 \mathrm{X}$

Fig.1 SEM images of $1 \#$ specimen

crack develop evenly. Specifically, it can be seen from macrograph of mudstone that the basic unit of the rock is blocky particles, the arrangement of particles has certain direction, the structure is a little loose with a few small holes, size distribution is not uniform and pore account for a small area. From the middle times picture we can see that: the size, shape, distribution of micropore is more obvious, the structure is relatively loose, there are honeycomb or mesh structures, secondary pore appear; local characteristics observed from high power figure in a certain individual show that massive and granular body and surface is blanketed with scaly and clay minerals, and some clay minerals stacked together to form larger particles. Small particles (clay minerals) fill in the larger particles, which acts as a binder. The clay minerals and the cementation material is small, the particles of skeleton role is large in size.

\subsection{Mudstone meso-structure surface fractal dimension calculation .}

MATLAB is a powerful scientific and engineering calculation software, which can combine image processing, matrix operations, numerical analysis, programming techniques and other several functions organically, as well as the rich visual performance[9]. The fractal dimension calculation of rock surface crack involves the content of the image processing and numerical calculation, which can more reflect its special advantages.

In the process of box dimension calculation, the image should be processed with MATLAB first, through gray processing, histogram adjustment, smooth filtering, sharpening filter and binary processing, the binary image can be obtained. The binary image can be seen as a $\mathrm{M} \times \mathrm{N}$ data matrix. It ranks number correspond to the binary image's , and each data value 1 or 0 , which depending on its corresponding point that is black or white, if it is black, the corresponding data is 0 ,otherwise, it is 1 . Then the data file is divided into several ranks and all its number is $\mathrm{k}$, calculate the 
number occupied by block comprising 1 in a matrix, and named it as $N\left(\delta_{k}\right)$. And then change the side length of box: take $\mathrm{k}$ $=1,2,4 \ldots, 2 \mathrm{i}$, that divide data file which is based on the size of $\mathrm{k}$ pixels, then get the corresponding box number $N\left(\delta_{k}\right)$. Let $\delta$ is a pixel size, the rows and columns are $\mathrm{k}$ box of length of $k \delta$. For a specific image, the $\delta$ is a constant, So in the actual calculation, the $k \delta$ can be directly replaced by $\mathrm{k}$, take the logarithm of the box number $N\left(\delta_{k}\right)$ and side length $k \delta$ that obtained, and obtained the linear fitting by $\ln N\left(\delta_{k}\right)$ and $\ln k \delta$, the absolute value of the straight line slope is the image's fractal dimension ${ }^{[10]}$. The process can be easily implemented in the environment of MATLAB.

The rock microscopic crack can be used to study by the fractal theory, for it has self similarity statistically, which is conformed to the characteristic of fractal theory. Calculating the fractal dimension can better reflect the development degree of rock on mesoscopic and distribution uniformity of rock crevices.

For a piece of rock image in terms of microscopic morphology, it can be divided into finite size, that is to say, when the $\delta$ at pixel scale, it is a two-dimensional set, and it no longer has the fractal properties. Therefore, when we discuss fractal image, it must restricted $\delta$ within a certain scale, at least not less than one pixel scale. Therefore, in the calculation of the SEM image fractal dimension, we will not take $\delta \rightarrow 0$, a set of discrete points in a proper range needed to be chosen, so as to calculate the corresponding number of boxes.

Verify the calculation method of the program with known regular fractal image like straight , rectangular, kochsnow curves. The results showed that, the calculated error is relatively small, which was within $5 \%$, so this result is reliable.

\section{Fractal Characteristics Analysis of Mudstone}

4.1 The fractal dimension of mudstone

The SEM image of mudstone specimens at 100 to 5000 times are as shown in Figure 1 (in the case of $1 \#$ ). For the mudstone specimen, the author processed the microstructure image and calculate its fractal dimension by the compiled program, the results are shown in table1.

Take specimen1 as an example, the binary image and fractal dimension are shown in Figure 3 and figure 4:

Tab. 1 the fractal dimension of three groups of mudstone

\begin{tabular}{|c|c|c|c|c|}
\hline & $1 \#$ & $2 \#$ & $3 \#$ & Average \\
\hline 100 & 2.1451 & 2.1425 & 2.1323 & 2.1400 \\
\hline 200 & 2.1125 & 2.1135 & 2.1034 & 2.1098 \\
\hline 500 & 2.0859 & 2.1067 & 2.0812 & 2.0913 \\
\hline 1000 & 2.0502 & 2.1031 & 2.0429 & 2.0654 \\
\hline 2000 & 2.0328 & 2.0933 & 2.0311 & 2.0524 \\
\hline 5000 & 2.0245 & 2.0766 & 2.0248 & 2.0420 \\
\hline Average & 2.0752 & 2.1060 & 2.0693 & 2.0835 \\
\hline
\end{tabular}




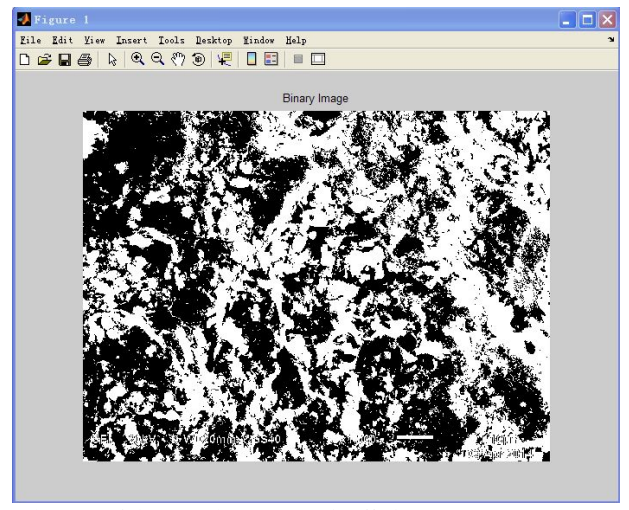

Fig.3 binary image of $1 \#$ for $1000 \mathrm{X}$

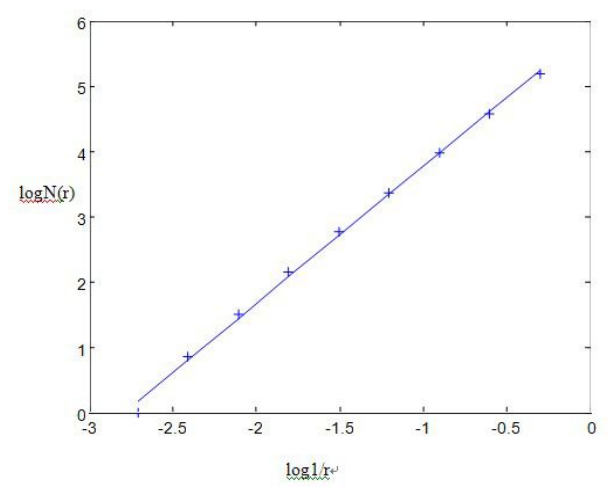

Fig. 4 fractal dimension of $1 \#$ for $1000 \mathrm{X}$

\subsection{Analysis of Fractal Characteristics}

Three groups of mudstone fractal dimension under different multiples are shown in Table 1. The results showed that marble showed good self-similarity. As we can see from the data in the table, the fractal dimension of different specimens are different. Different fractal dimensions show that their complexity and roughness are different. Among them, texture of Specimen 1 is monotonous and rough, texture of Specimen 3 is dense and in great regularity. Both of their surface morphology are relatively simple, so their fractal dimension are less and similar $(D=2.0752$ and $D=2.0693)$; but fractal dimension of Specimen 2 is larger than them obviously. Though texture of Specimen 2 is Simple and smooth, it has less regularity and is rougher, so its fractal dimension is larger $(D=2.1060)$. With the increase of SEM scanning multiple, based on its self-similarity, the overall trend of the fractal dimension is reduced. When under the $100 \mathrm{X}$, the fractal dimension is the highest, surface appearance is the most complex; When under the $5000 \mathrm{X}$, the fractal dimension is the lowest. Generally speaking, with the increase of multiple, the overall trend of its fractal dimension increases.

\section{Conclusions}

The above analysis and calculations showed that: Mudstone microstructure has self-similarity in statistic, the fractal dimension obtained by box counting dimension can quantitatively describes complexity and irregular behavior of the surface texture structure. Mudstone microstructure inside determines stress strain state under the external force, which controls the macroscopic mechanical failure mechanism.

\section{Reference:}

[1] Yunliang Tan, Chuanxiao Liu, Tongbin Zhao. Initial Exploration into Nonlinear Dynamics Rocks[M]. Beijing: Coal Industry Press. 2007

[2] Xiaopeng $\mathrm{Xu}$, Rundong Peng and others, Research on evolution method of Fractal dimension estimation of brittle material microstructure based on SEM images. Rock Mechanics and Engineering Learned Journal. 23(21): 3600 3603

[3] Jinzhao Zhu, Qingke Zhu. The research progress of calculation methodof Fractal Dimension[J]. Beijing Forestry University Learned Journal,2002,24(2):72-28.

[4] Feng Gao,Heping Xie,Peng Zhao.The fractal nature of the distribution of the block and Microstructure effects of rock.Rock Mechanics and Engineering Learned Journal, 1994,13(3):240.

[5] Ruidong Peng, Heping Xie, Yang Ju.Fractal dimension calculation method of two-dimensional digital image[J].China University of Mining \& Technology Learned Journal,2004,33(1):19-24. 
[6]Feranie S , Fauzi U,Bijaksana S. 3D fractal dimension and flow properties in the pore struture of geological rocks $[\mathrm{J}]$. Fractuals, 2011.19(3):291-297.

[7] Jiande Li, Xuena Jia, Jinli Miao and others. The fractal characteristic analysis of scrap of granite rock burst test[J]. Rock Mechanics and Engineering Learned Journal, 2010,29(1):3280-3289. [8] Jinghong Liu, Yaodong Jiang, Yixin Zhao and others.Analysis of rock failure process fractal characteristics of crack based on CT images[J]. Agricultural
University of Hebei Learned Journal , 2011,34(4):104-107.

[9] Haitao Li,Ying Deng. MATLAB

Programming Guide[M]. Beijing:Higher Education Press, (2002),2004,18(4).

[10] Liguo Xiao,Cheng Zhong.An improved fractal image compression algorithm and its complexity Analysis[J].Guangxi University Learned Journal(Natural Science), 2002, 4 (27): 330-333. 